\title{
FIGO Stage II Gestational Trophoblastic
} Tumor

National Cancer Institute

\section{Source}

National Cancer Institute. FlGO Stage II Gestational Trophoblastic Tumor. NCI

Thesaurus. Code C6396.

Lesion invades outside of the uterus, but it is limited to the genital structures. 